\title{
TWISTED GROUP RINGS WHICH ARE SEMI-PRIME GOLDIE RINGS
}

\author{
by A. REID $\dagger$ \\ (Received 6 October, 1973; revised 13 March, 1974)
}

In this paper we examine when a twisted group ring, $R^{\gamma}(G)$, has a semi-simple, artinian quotient ring. In $\S 1$ we assemble results and definitions concerning quotient rings, Ore sets and Goldie rings and then, in $\S 2$, we define $R^{\gamma}(G)$. We prove a useful theorem for constructing a twisted group ring of a factor group and establish an analogue of a theorem of Passman. Twisted polynomial rings are discussed in $\$ 3$ and I am indebted to the referee for informing me of the existence of [4]. These are used as a tool in proving results in $\$ 4$.

A group $G$ is a poly- (torsion-free abelian or finite) group if $G$ has a series of subgroups $\{e\}=H_{0} \triangleleft H_{1} \triangleleft H_{2} \triangleleft \ldots \triangleleft H_{n}=G$ such that $H_{i} / H_{i-1}$ is either torsion-free abelian or finite $(i=1,2, \ldots, n)$. These groups are considered here and we prove (Theorem 4.5) that if such a group $G$ has only a finite set $S$ of periodic elements with $|S|$ regular in $R$ and $R$ is semi-prime, left Goldie, then $R^{\gamma}(G)$ is semi-prime, left Goldie.

In $\S 5$ we define a class of groups $\mathscr{C}$ such that if $G$ is a torsion-free element of $\mathscr{C}$ and $D$ is a division ring then $D^{\gamma}(G)$ is an Ore domain. We call these groups Ore groups and prove a theorem similar to Theorem 4.5 for this class of groups.

Throughout, $R$ will denote a ring with identity element 1 and $G$ a multiplicative group with identity $e$. By artinian and noetherian we mean left artinian and left noetherian.

\section{Goldie rings.}

We restate the following definitions which appear in [2, pp. 228, 229].

An element of a ring $R$ is regular if it is neither a left nor a right zero divisor. A set $T$ of regular elements of $R$ which is multiplicatively closed is a left Ore set if, whenever $a \in R$, $c \in T$, there exist $a^{\prime} \in R, c^{\prime} \in T$ such that $c^{\prime} a=a^{\prime} c$.

A ring $Q$ is a left quotient ring of $R$ with respect to a set $T$ of regular elements of $R$ if

(i) $Q \supseteq R$,

(ii) the elements of $T$ are units in $Q$,

(iii) the elements of $Q$ have the form $c^{-1} a$ where $c \in T, a \in R$.

If such a ring $Q$ exists, it will be denoted by $R_{T}$. When $T$ is the set of all regular elements of $R$ we say that $Q$ is the left quotient ring of $R$.

THEOREM 1.1. Let $T$ be a set of regular elements of $R$. Then $R_{T}$ exists if and only if $T$ is a left Ore set in $R$.

$\dagger$ This work was supported by the Science Research Council and forms part of the author's Ph.D. thesis (Aberdeen). I wish to thank Professor D. A. R. Wallace for his invaluable encouragement and advice and the referee for his helpful suggestions. In particular $I$ am indebted to the referee for pointing out the method of proof of Theorem 2.7. My original, longer proof followed the lines of [6, Appendices 2, 3]. 
Proof. [3, p. 170].

A ring $R$ has finite left Goldie rank if it contains no infinite direct sum of non-zero left ideals. Let $S$ be a non-empty subset of $R$; then $\ell(S)$, the left annihilator of $S$, is the left ideal $\{a \in R: a s=0$ for all $s \in S\}$. A ring $R$ is a left Goldie ring if (i) $R$ has finite left Goldie rank and (ii) $R$ has ascending chain condition on left annihilators.

GoLdIE's THEorem [2, Theorem 1.37]. A ring $R$ has a semi-simple artinian left quotient ring if and only if $R$ is a semi-prime left Goldie ring.

LEMMA $1.2 \quad[11$, Corollary 2.5]. Let $Q$ be an artinian ring with subring $R$ such that every element of $Q$ has the form $c^{-1} a$, where $c, a \in R$. Then $Q$ is the left quotient ring of $R$.

For convenience, we formulate the following straightforward lemmas.

LEMMA 1.3. Let $R$ be a ring and let $T \subseteq R$ be a left Ore set.

(i) Let $L$ be a left ideal and let $L_{T}=R_{T} L$, the left ideal in $R_{T}$ generated by $L$. Then $L_{T}=\left\{c^{-1} r: c \in T, r \in L\right\}$.

(ii) Let $L$ and $J$ be left ideals in $R$. Then $L_{T} \cap J_{T}=(L \cap J)_{T}$.

(iii) If $L$ is a left annihilator in $R$, then $L_{T}$ is a left annihilator in $R_{T}$ and $L_{T} \cap R=L$.

(iv) If $R_{T}$ is a left Goldie ring, then $R$ is a left Goldie ring.

Lemma 1.4. Let $R_{1}, R_{2}, \ldots, R_{n}$ be a finite number of left Goldie rings. Then $R=$ $R_{1} \oplus R_{2} \oplus \ldots \oplus R_{n}$ is also a left Goldie ring.

\section{Twisted group rings.}

Definition. Let $G$ be a group with identity element $e, R$ a ring with identity $1, R^{*}$ the group of central units of $R$ and $\gamma: G \times G \rightarrow R^{*}$ a 2-cocycle. [That is, $\gamma(g, h) \gamma(g h, k)=$ $\gamma(g, h k) \gamma(h, k), g, h, k \in G]$. Let $R^{\gamma}(G)$ be the free left $R$-module with basis $\{\bar{g}: g \in G\}$. Define multiplication in $R^{\gamma}(G)$ by

$$
\bar{g} \bar{h}=\gamma(g, h) \overline{g h} \quad(g, h \in G)
$$

extending this, by linearity, to the whole of $R^{\gamma}(G)$. Then $R^{\gamma}(G)$ is an associative ring with identity element $\gamma(e, e)^{-1} \bar{e}$. We call $R^{\gamma}(G)$ the $t$ wisted group ring of $G$ over $R$ with $t w i s t \gamma$.

We shall identify an element $r \in R$ with its image $r \gamma(e, e)^{-1} \bar{e}$ in $R^{\gamma}(G)$.

In this section we prove some results about $R^{\gamma}(G)$ that we shall require later.

THEOREM 2.1. Let $G$ be a group with a central normal subgroup $Z$ and $R^{\gamma}(G)$ a twisted group ring such that $\gamma(g, z)=\gamma(z, g)$ for all $g \in G$ and $z \in Z$. Then there exists a twisted group ring of $G / Z$ over $R^{\gamma}(Z)$ with twist $\delta$ such that

$$
R^{\gamma}(G) \cong\left[R^{\gamma}(Z)\right]^{\delta}(G / Z)
$$


Proof. Let $T$ be a set of coset representatives for $Z$ in $G$. Then every element of $G$ is uniquely represented in the form $t z$ for some $t \in T, z \in Z$. Thus given $t_{1}, t_{2} \in T$ there are a unique $\tau\left(t_{1}, t_{2}\right) \in T$ and $z \in Z$ such that $t_{1} t_{2}=\tau\left(t_{1}, t_{2}\right) z$. Then, in $R^{\gamma}(G)$,

$$
t_{1} \bar{t}_{2}=\gamma\left(t_{1}, t_{2}\right) \overline{\tau\left(t_{1}, t_{2}\right) z}=\gamma\left(t_{1}, t_{2}\right) \gamma\left(z, \tau\left(t_{1}, t_{2}\right)\right)^{-1} \bar{z} \overline{\tau\left(t_{1}, t_{2}\right)} .
$$

Thus

$$
i_{1} \tau_{2}\left(\overline{\left(\tau\left(t_{1}, t_{2}\right)\right.}\right)^{-1}=\gamma\left(t_{1}, t_{2}\right) \gamma\left(z, \tau\left(t_{1}, t_{2}\right)\right)^{-1} \bar{z} \in \text { central units of } R^{\gamma}(Z) .
$$

Let $F=G / Z$. Then for each $f \in F$ there is a unique $t \in T$ such that $f=t Z$. Define $\delta: F \times F \rightarrow$ $\left(R^{\gamma}(Z)\right)^{*}$ by

$$
\delta\left(f_{1}, f_{2}\right)=\bar{t}_{1} \bar{t}_{2}\left(\overline{\tau\left(t_{1}, t_{2}\right)}\right)^{-1}, \text { where } f_{1}=t_{1} Z, f_{2}=t_{2} Z, t_{1}, t_{2} \in T .
$$

Given $f_{1}, f_{2}$, then $t_{1}, t_{2}$ and $\tau\left(t_{1}, t_{2}\right)$ are uniquely determined. Thus $\delta$ is well-defined and it is readily verified that $\delta$ is a 2 -cocycle.

Hence we have defined $\left[R^{\gamma}(Z)\right]^{\delta}(F)$. We shall denote by $f^{*}$ the image in $\left[R^{\gamma}(Z)\right]^{\delta}(F)$ of an element $f \in F$.

Now we construct an isomorphism between $R^{\gamma}(G)$ and $\left[R^{\gamma}(Z)\right]^{\delta}(F)$. As remarked earlier, given $g \in G$ there are a unique $t \in T$ and $z \in Z$ with $g=t z=z t$. Then $\bar{g}=\gamma(z, t)^{-1} \bar{z} \bar{t}$ in $R^{\gamma}(G)$. Define $\theta: R^{\gamma}(G) \rightarrow\left[R^{\gamma}(Z)\right]^{\delta}(F)$ to be the $R$-homomorphism defined by

$$
\theta: \bar{g}=\gamma(z, t)^{-1} \bar{z} \bar{t} \mapsto \gamma(z, t)^{-1} \bar{z}(t Z)^{*} .
$$

We show that $\theta$ is also a ring homomorphism. To do this, it is sufficient to show that $\theta\left(\bar{g}_{1} \bar{g}_{2}\right)=$ $\theta\left(\bar{g}_{1}\right) \theta\left(\bar{g}_{2}\right)\left(g_{1}, g_{2} \in G\right)$. Let $g_{1}=z_{1} t_{1}, g_{2}=z_{2} t_{2}$, where $z_{1}, z_{2} \in Z, t_{1}, t_{2} \in T$. Then

$$
\begin{aligned}
\bar{g}_{1} \bar{g}_{2} & =\gamma\left(z_{1}, t_{1}\right)^{-1} \bar{z}_{1} \bar{t}_{1} \gamma\left(z_{2}, t_{2}\right)^{-1} \bar{z}_{2} \bar{t}_{2} \\
& =\gamma\left(z_{1}, t_{1}\right)^{-1} \gamma\left(z_{2}, t_{2}\right)^{-1} \gamma\left(z_{1}, z_{2}\right) \gamma\left(t_{1}, t_{2}\right) \overline{z_{1} z_{2}} \overline{t_{1} t_{2}} \\
& =\gamma\left(z_{1}, t_{1}\right)^{-1} \gamma\left(z_{2}, t_{2}\right)^{-1} \gamma\left(z_{1}, z_{2}\right) \gamma\left(z_{1} z_{2}, z_{3}\right) \gamma\left(z_{3}, t_{3}\right)^{-1} \gamma\left(t_{1}, t_{2}\right) \overline{z_{1} z_{2} z_{3}} \overline{t_{3}}
\end{aligned}
$$

(where $t_{1} t_{2}=z_{3} t_{3}, z_{3} \in Z, t_{3} \in T$ ). Thus

$$
\theta\left(\bar{g}_{1} \bar{g}_{2}\right)=\gamma\left(z_{1}, t_{1}\right)^{-1} \gamma\left(z_{2}, t_{2}\right)^{-1} \gamma\left(z_{1}, z_{2}\right) \gamma\left(z_{1} z_{2}, z_{3}\right) \gamma\left(z_{3}, t_{3}\right)^{-1} \gamma\left(t_{1}, t_{2}\right) \overline{z_{1} z_{2} z_{3}}\left(t_{3} Z\right)^{*} .
$$

Also

$$
\begin{aligned}
\theta\left(\bar{g}_{1}\right) \theta\left(\bar{g}_{2}\right) & =\gamma\left(z_{1}, t_{1}\right)^{-1} \bar{z}_{1}\left(t_{1} Z\right)^{*} \gamma\left(z_{2}, t_{2}\right)^{-1} \bar{z}_{2}\left(t_{2} Z\right)^{*} \\
& =\gamma\left(z_{1}, t_{1}\right)^{-1} \gamma\left(z_{2}, t_{2}\right)^{-1} \gamma\left(z_{1}, z_{2}\right) \overline{z_{1} z_{2}} \delta\left(t_{1} Z, t_{2} Z\right)\left(t_{3} Z\right)^{*} .
\end{aligned}
$$

Thus, recalling that

$$
\delta\left(t_{1} Z, t_{2} Z\right)=\bar{t}_{1} \bar{Z}_{2}\left(\bar{t}_{3}\right)^{-1}=\gamma\left(t_{1}, t_{2}\right) \gamma\left(z_{3}, t_{3}\right)^{-1} \bar{z}_{3}
$$

it follows that $\theta\left(\bar{g}_{1} \bar{g}_{2}\right)=\theta\left(\bar{g}_{1}\right) \theta\left(\bar{g}_{2}\right)$. 
Hence $\theta$ is a ring homomorphism and, since $\theta$ is clearly both one-one and onto, the required isomorphism is established.

COROLlaRY 2.2. Let $G$ be a group, $Z$ a central normal subgroup of $G$ and $R$ a ring. Then there exists a twisted group ring of $G / Z$ over $R(Z)$ with twist $\delta$, such that

$$
R(G) \cong R(Z)^{8}(G / Z)
$$

Thus twisted group rings occur in a fairly natural way and we have a useful method of expressing a group ring in terms of a subgroup and a factor group.

For Lemma 2.5 we shall require the following result. We denote the set of positive integers by $\mathbf{P}$.

Lemma 2.3. Let $R$ be a semi-simple, artinian ring and let $n \in \mathbf{P}$. Let $W=\left\{w \in R^{*}\right.$ : $\left.w^{n}=1\right\}$. Then $W$ is finite.

Proof. Let $S$ be the centre of $R$. Then, since $R$ is semi-simple artinian, there exist fields $F_{1}, F_{2}, \ldots, F_{r}$ (say) such that $S=F_{1} \oplus F_{2} \oplus \ldots \oplus F_{r}$. For $w \in W$, let $\left(w_{1}, w_{2}, \ldots, w_{r}\right)$ be the image of $w$ in $F_{1} \oplus F_{2} \oplus \ldots \oplus F_{r}$. Then $w^{n}=1$ implies that $w_{i}^{n}=1(i=1,2, \ldots, r)$. Hence $W=W_{1} \oplus W_{2} \oplus \ldots \oplus W_{r}$, where $W_{l}$ is the set of $n$th roots of unity in $F_{i}$. But the set of $n$th roots of unity in a field is finite. Hence $W$ is finite.

COROLlaRY 2.4. Let $R$ be a semi-prime left Goldie ring and let $n \in \mathbf{P}$. Let $W=\left\{w \in R^{*}\right.$ : $\left.w^{n}=1\right\}$. Then $W$ is finite.

Proof. Let $Q$ be the semi-simple, artinian quotient ring of $R$. Then $W \subseteq\left\{w \in Q^{*}\right.$ : $\left.w^{n}=1\right\}$ which, by the lemma, is finite.

Definition. Let $R^{\gamma}(G)$ be a twisted group ring and let $H \leqq G$. Define

$$
\begin{aligned}
\bar{C}_{G}(H) & =\{g \in G: \bar{g} \bar{h}=\bar{h} \bar{g} \text { for all } h \in H\} \\
& =\left\{g \in C_{G}(H): \gamma(g, h)=\gamma(h, g) \text { for all } h \in H\right\} .
\end{aligned}
$$

It is readily verified that $\bar{C}_{G}(H)$ is a subgroup of $G$.

Lemma 2.5. Let $R$ be a semi-prime left Goldie ring and let $R^{\gamma}(G)$ be a twisted group ring. Let $H$ be a subgroup of $G$. Then $(i) \bar{C}_{G}(H) \triangleleft C_{G}(H)$ and (ii) if, further, $|H|<\infty$, then $\left|C_{G}(H): \bar{C}_{G}(H)\right|<\infty$.

Proof. Let $g_{1}, g_{2} \in C_{G}(H), h \in H$. Then

$$
\frac{\gamma\left(g_{1}, h\right) \gamma\left(g_{2}, h\right)}{\gamma\left(h, g_{1}\right) \gamma\left(h, g_{2}\right)}=\frac{\gamma\left(g_{1}, h\right) \gamma\left(g_{2}, h\right) \gamma\left(h g_{1}, g_{2}\right)}{\gamma\left(h, g_{1}\right) \gamma\left(h, g_{2}\right) \gamma\left(h g_{1}, g_{2}\right)}
$$


Now define $\theta_{h}: C_{G}(H) \rightarrow R^{*}$ by

$$
\begin{aligned}
& =\frac{\gamma\left(g_{1}, h g_{2}\right) \gamma\left(h, g_{2}\right) \gamma\left(g_{2}, h\right)}{\gamma\left(h, g_{1} g_{2}\right) \gamma\left(g_{1}, g_{2}\right) \gamma\left(h, g_{2}\right)} \\
& =\frac{\gamma\left(g_{1}, g_{2}\right) \gamma\left(g_{1} g_{2}, h\right)}{\gamma\left(h, g_{1} g_{2}\right) \gamma\left(g_{1}, g_{2}\right)} \\
& =\gamma\left(g_{1} g_{2}, h\right) \gamma\left(h, g_{1} g_{2}\right)^{-1}
\end{aligned}
$$

$$
\theta_{h}(g)=\gamma(g, h) \gamma(h, g)^{-1} \quad\left(g \in C_{G}(H)\right) .
$$

Then, by the above argument, $\theta_{h}$ is a group homomorphism, $\operatorname{Ker} \theta_{h}=\left\{g \in C_{G}(H): \gamma(g, h)=\right.$ $\gamma(h, g)\}$ and hence

$$
\bar{C}_{G}(H)=\bigcap_{h \in H} \operatorname{Ker} \theta_{h}
$$

It follows that $\bar{C}_{G}(H) \triangleleft C_{G}(H)$.

Now suppose that $|H|=n$ and let $h \in H, g \in C_{G}(H)$. Then $(h \bar{g})^{n}=a h^{n} \bar{g}^{n}$ for some $a \in R$. But $h^{n}=e$ therefore $h^{n} \in R$ and so $(h \bar{g})^{n}=b \bar{g}^{n}$ for some $b \in R$. Thus

$$
(\bar{h} \bar{g})^{n}=\bar{g}(\bar{h} \bar{g})^{n} \bar{g}^{-1}=(\bar{g} \bar{h})^{n}=\left[\gamma(g, h) \gamma(h, g)^{-1} \bar{h} \bar{g}\right]^{n} .
$$

Therefore $\left[\gamma(g, h) \gamma(h, g)^{-1}\right]^{n}=1$ and so

$$
C_{G}(H) / \operatorname{Ker} \theta_{h} \cong \text { subgroup of group of } n \text {th roots of unity in } R^{*} .
$$

Hence, by Corollary 2.4, $\left|C_{G}(H): \operatorname{Ker} \theta_{h}\right|<\infty$. Further, since $|H|<\infty,\left|C_{G}(H): \bar{C}_{G}(H)\right|<\infty$ and the result is proved.

We now give a lemma concerning rings of quotients.

LEMMA 2.6. (i) Let $H \triangleleft G$ such that $R^{\gamma}(G)$ has a left quotient ring and let $T$ be the set of regular elements in $R^{\gamma}(H)$. Then $T$ is a left Ore set in $R^{\gamma}(G)$.

(ii) If $R$ has a left quotient ring $Q$, then $Q^{\gamma}(G)$ is well-defined and is the left quotient ring of $R^{r}(G)$ with respect to the set of regular elements of $R$.

Proof. (i) Adapt [12, Lemma 2.6].

(ii) This is clear.

We shall wish to know when $R^{\gamma}(G)$ is semi-prime. We denote by $P R^{\gamma}(G)$ the prime radical of $R^{\gamma}(G)$. In the 'untwisted' situation we have the following theorem due to D. Passman $[6$, p. 162, see also 7] and I. Connell [6, Appendices 2 and 3].

THEOREM A. The group ring $R(G)$ is semi-prime if and only if $R$ is semi-prime and the order of each finite normal subgroup of $G$ is regular in $R$.

In [8, Theorem 3.7] Passman proves the following extension of this. 
THEOREM B. Let $K$ be an algebraically closed field of characteristic $p>0$ and $K^{\gamma}(G) a$ twisted group ring. Then $K^{\gamma}(G)$ is semi-prime if and only if $G$ has no finite normal subgroups of order divisible by $p$.

Let $K$ be any field of characteristic $p>0, F$ its algebraic closure and $K^{\gamma}(G)$ a twisted group ring. Then $F^{\gamma}(G)$ is well-defined and, arguing as in [1, Proposition 9], it can be shown that

$$
P K^{\gamma}\left(G=K^{\gamma}(G) \cap P F^{\gamma}(G) .\right.
$$

It is immediate from this and Theorem B that, if $G$ has no finite normal subgroups of order divisible by $p$ then, $K^{\gamma}(G)$ is semi-prime and we generalise this below in Theorem 2.7. The converse of this, however, is not true. We recall a counter example discussed in [9]. Let $K$ be a field over which the polynomials $x^{p^{n}}-a$ are irreducible for some $a \in K$ and where $p=$ char $K$. Let $G=\mathrm{Z}_{p}{ }^{\infty}$. Then we may construct a twisted group ring $K^{\gamma}(G)$ which is a field and hence semi-prime. The orders of finite normal subgroups of $G$, however, are powers of $p$.

THEOREM 2.7. Let $R$ be a semi-prime ring and one of the following: (i) commutative, (ii) a semi-direct product of simple rings, (iii) left Goldie. Let $G$ be a group such that the order of each finite normal subgroup is regular in $R$ and let $R^{\gamma}(G)$ be a twisted group ring. Then $R^{\gamma}(G)$ is semi-prime.

Proof. (i) As in [1, proof of Theorem 5, p. 668].

(ii) As in [1, proof of Proposition 10, pp. 669 and 670].

(iii) Let $Q$ be the semi-simple artinian left quotient ring of $R$. Then, by (ii), $Q^{\gamma}(G)$ is semi-prime and hence $R^{\gamma}(G)$ is semi-prime.

\section{Twisted polynomial rings.}

Definition. Let $R$ be a ring and $\theta: R \rightarrow R$ an automorphism of $R$. Let $\langle x\rangle$ be an infinite cyclic group. We define $R_{\theta}(x)$ to be the free left $R$-module with basis $\langle x\rangle$ and, for $r \in R$, we define multiplication on $R_{\theta}(x)$ by

$$
\begin{aligned}
x r & =\theta(r) x \\
x^{-1} r & =\theta^{-1}(r) x^{-1},
\end{aligned}
$$

extending by linearity to the whole of $R_{\theta}(x)$. With this definition of multiplication $R_{\theta}(x)$ is an associative ring.

Thus $R_{\theta}(x)$ is a ring of polynomials in $x$ and $x^{-1}$ with coefficients from $R$. The subring of $R_{\theta}(x)$ containing only the polynomials in non-negative powers of $x$, denoted by $R_{\theta}[x]$, is called a twisted polynomial ring.

A. Horn in $[4, \S 2]$ has proved the following.

THEOREM 3.1. Let $R$ be a noetherian ring. Then $R_{\theta}[x]$ has an artinian left quotient ring if and only if $R$ has an artinian left quotient ring. 
From this we may deduce the following corollary.

COROLlaRY 3.2. Let $R$ have an artinian left quotient ring. Then $R_{\theta}(x)$ has an artinian left quotient ring.

Proof. Let $Q$ be the left quotient ring of $R$. Then, by the theorem, $Q_{\theta}[x]$ has an artinian left quotient ring $\bar{Q}$. Since $x^{i}$ is regular in $Q_{\theta}[x], x^{-i} \in \bar{Q}(i \in \mathbf{P})$ and hence

$$
R_{\theta}(x) \subseteq Q_{\theta}(x) \subset \bar{Q} .
$$

It is now clear from Lemma 1.2 that $\bar{Q}$ is the artinian left quotient ring of $R_{\theta}(x)$.

4. Quotient rings of $R^{\gamma}(G)$. In this section we obtain sufficient conditions for $R^{\gamma}(G)$ to have a semi-simple artinian quotient ring, similar to but less stringent than those obtained by P. Smith in [12, Theorem 2.18] for $R(G)$. By Goldie's Theorem, if $R^{\gamma}(G)$ is to have a semisimple artinian left quotient ring, then it must itself be a semi-prime left Goldie ring and therefore must have both a.c.c. on left annihilators and finite left Goldie rank.

Lemma 4.1. Let $R^{\gamma}(G)$ be semi-prime and let $H \triangleleft G$ be such that (i) $|G: H|<\infty$ and (ii) $R^{\gamma}(H)$ is semi-prime left Goldie. Then $R^{\gamma}(G)$ is semi-prime left Goldie.

Proof. By Lemma 2.6, the set $T$ of regular elements of $R^{\gamma}(H)$ is a left Ore set in $R^{\gamma}(G)$. Let $S=\left[R^{\gamma}(G)\right]_{T}$. Then $S$ is semi-prime and $S=\sum_{c \in C} Q \bar{c}$, where $Q$ is the left quotient ring of $R^{\gamma}(H)$ and $C$ is a set of coset representatives for $H$ in $G$. But $C$ is finite; therefore $S$ is an artinian $Q$-module and hence an artinian ring. It follows from Lemma 1.2 that $S$ is the left quotient ring of $R^{\gamma}(G)$ and so, by Goldie's Theorem, $R^{\gamma}(G)$ is a semi-prime left Goldie ring.

LemmA 4.2. Let $R^{\gamma}(G)$ have a left quotient ring and let $H \triangleleft G$ be such that

(i) $R^{\gamma}(H)$ is semi-prime left Goldie, and

(ii) $G / H$ is ordered.

Then $R^{\gamma}(G)$ is semi-prime left Goldie.

Proof. We prove that every essential left ideal in $R^{\gamma}(G)$ contains a regular element. Let $E$ be an essential left ideal in $R^{\gamma}(G)$ and let

$$
\begin{aligned}
E_{0}= & \left\{a \in R^{\gamma}(H): \bar{g}_{0} a+\bar{g}_{1} a_{1}+\ldots+\bar{g}_{n} a_{n} \in E \text { for some } n \text { and } a_{i} \in R^{\gamma}(H)\right. \text { and where } \\
& \left.g_{0} H<g_{1} H<\ldots<g_{n} H \text { in } G / H\right\} .
\end{aligned}
$$

Then $E_{0}$ is a left ideal in $R^{\gamma}(H)$. Let $a \in R^{\gamma}(H), a \neq 0$. Then there exists $\alpha=k_{1} b_{1}+k_{2} b_{2}+$ $\ldots+k_{m} b_{m} \in R^{\gamma}(G), b_{i} \in R^{\gamma}(H), k_{1} H<k_{2} H<\ldots<k_{m} H$ in $G / H$, such that $\alpha a \neq 0$ and $\alpha a \in E$. Therefore $b_{i} a \neq 0$ and $b_{i} a \in E_{0}$ for some $1 \leqq i \leqq m$ and it follows that $E_{0}$ is essential in $R^{\gamma}(H)$. But $R^{\gamma}(H)$ is semi-prime left Goldie; therefore $E_{0}$ contains a regular element of 
$R^{\gamma}(H)$. That is, there exists $x \in E$ with $x=\bar{g}_{0} c+\bar{g}_{1} c_{1}+\ldots+\bar{g}_{n} c_{n}$, where $\left.c_{i} \in R^{\gamma}(H)\right), c$ is regular in $R^{\gamma}(H)$ and $g_{0} H<g_{1} H<\ldots<g_{n} H$ in $G / H$. It is readily verified that $x$ is regular in $R^{\gamma}(G)$.

Now since every essential left ideal of $R^{\gamma}(G)$ contains a regular element, $Q$, the left quotient ring of $R^{\gamma}(G)$, contains no proper essential left ideals and is therefore a semi-simple artinian ring [2, p. 234 and p. 219].

Corollary 4.3. Let $R^{\gamma}(G)$ be a twisted group ring and $H \triangleleft G$ be such that $G / H$ is infinite cyclic and $R^{\gamma}(H)$ is semi-prime left Goldie. Then $R^{\gamma}(G)$ is semi-prime left Goldie.

Proof. $G / H=\langle g H\rangle$ for some $g \in G \mid H$. Define $\theta: R^{\gamma}(H) \rightarrow R^{\gamma}(H)$ by $\theta(\alpha)=\bar{g} \alpha \bar{g}^{-1}$ $\left(\alpha \in R^{\gamma}(H)\right)$. Then, since $H \triangleleft G, \theta$ is an automorphism of $R^{\gamma}(H)$ and, in the notation of $\S 3$, with $\bar{g}=x, R^{\gamma}(G)=R^{\gamma}(H)_{\theta}(\bar{g})$. Now it follows from Corollary 3.2 that $R^{\gamma}(G)$ has an artinian left quotient ring and so, $G / H$ being an ordered group, $R^{\gamma}(G)$ is semi-prime left Goldie.

Lemma 4.4. Let $R^{\gamma}(G)$ be a twisted group ring and let $H \triangleleft G$ be such that (i) $R^{\gamma}(H)$ is semi-prime left Goldie, and (ii) $G / H$ is torsion-free abelian. Then $R^{\gamma}(G)$ is semi-prime left Goldie.

Proof. $G / H$ is an ordered group. Thus, from Lemma 4.2, it will be sufficient to prove that $R^{\gamma}(G)$ has a left quotient ring. To do so it is enough to show that $R^{\gamma}\left(G_{1}\right)$ has a left quotient ring for every subgroup $G_{1}$ such that $G_{1} / T$ is finitely generated. But $G_{1} / H$ is a direct sum of a finite number of infinite cyclic groups and the required result follows by induction from Corollary 4.3.

THEOREM 4.5. Let $G$ be a poly- (torsion-free abelian or finite) group and let $S$ be the set of all periodic elements of $G$. Let $R$ be semi-prime left Goldie and let $S$ be finite with $|S|$ regular in $R$. 'Then $R^{\gamma}(G)$ is semi-prime left Goldie.

Proof. By Theorem 2.7, $R^{\gamma}(G)$ is semi-prime and so the result follows by induction from Lemmas 4.1, 4.4.

EXAMPLES of poly-(torsion-free abelian or finite) groups.

(i) Nilpotent groups with finite set of periodic elements. (A torsion-free nilpotent group has central series with factors all torsion-free abelian [5, Theorem 1.2].)

(ii) Soluble groups with derived series whose factors have only a finite number of periodic elements.

(iii) $F C$-soluble groups $[10, \mathrm{pp} .121,129]$ with series

$$
\{e\}=H_{0} \triangleleft H_{1} \triangleleft \ldots \triangleleft H_{n}=G
$$

such that $H_{i+1} / H_{i}$ is an $F C$-group whose torsion subgroup [10, p. 121, Theorem 4.32] is finite $(i=0,1, \ldots, n-1)$.

((i) and (ii) are particular examples of (iii).) 


\section{Ore groups.}

DEFINITION. A ring $R$ is called a left Ore domain if

(i) $R$ contains no proper zero divisors, and

(ii) $R$ satisfies the left Ore condition.

We shall be interested in the class of groups such that, given $G$ torsion-free and an Ore domain $R$, then $R^{\gamma}(G)$ is an Ore domain. We therefore make the following definition.

DEFINITION. Let $\mathscr{C}$ be the class of groups such that

(i) $G \in \mathscr{C}, H \leqq G \Rightarrow H \in \mathscr{C}$,

(ii) $G \in \mathscr{C}, H \triangleleft G,|H|<\infty \Rightarrow G / H \in \mathscr{C}$,

(iii) if $G \in \mathscr{C}$ is torsion-free, $D$ is a division ring and $D^{\gamma}(G)$ a twisted group ring, then $D^{\gamma}(G)$ is an Ore domain.

If $G \in \mathscr{C}$ we call $G$ an Ore group. Every periodic group is an Ore group. Also abelian groups, nilpotent groups and $F C$-groups are Ore groups.

THEOREM 5.1. Let $G$ be a group such that any twisted group ring $D^{\delta}(G)$, where $D$ is a division ring, is semi-prime left Goldie. Let $R$ be a semi-prime left Goldie ring. Then $R^{\gamma}(G)$ is semi-prime left Goldie.

Proof. Let $Q$ be the semi-simple artinian quotient ring of $R$. By Lemmas 2.6 and 1.3, (iv), it is sufficient to prove that $Q^{\gamma}(G)$ is semi-prime left Goldie. Then

$$
Q=M_{n_{1}}\left(D_{1}\right) \oplus M_{n_{2}}\left(D_{2}\right) \oplus \ldots \oplus M_{n_{r}}\left(D_{r}\right)
$$

for some integers $n_{1}, \ldots, n_{r}$ and division rings $D_{1}, D_{2}, \ldots, D_{r}$. Also there exist orthogonal central idempotents $e_{1}, e_{2}, \ldots, e_{r} \in Q$ such that $M_{n_{i}}\left(D_{i}\right)=Q e_{i}(i=1,2, \ldots, r)$. Let $g, h \in G$. Since $\gamma(g, h)$ is a central unit of $R, \gamma(g, h) e_{i}$ is a central unit of $D_{i}(i=1,2, \ldots, r)$ and thus, defining $\gamma_{i}(g, h)=\gamma(g, h) e_{i}$, we have defined twisted group rings $D_{i}^{\gamma_{i}}(G)(i=1,2, \ldots, r)$. It follows that

$$
Q^{\gamma}(G)=M_{n_{1}}\left(D_{1}^{\gamma_{1}}(G)\right) \oplus M_{n_{2}}\left(D_{2}^{\gamma_{2}}(G)\right) \oplus \ldots \oplus M_{n_{r}}\left(D_{r}^{\gamma_{r}}(G)\right) .
$$

Hence it is sufficient to prove that each $M_{n_{l}}\left(D_{i}^{y_{1}}(G)\right)$ is semi-prime left Goldie. But $D_{i}^{\gamma_{1}}(G)$ has a semi-simple artinian quotient ring $Q_{i}$, by the hypotheses of the theorem; hence [11, Theorem 3.1] $M_{n_{i}}\left(Q_{t}\right)$ is the semi-simple artinian quotient ring of $M_{n_{t}}\left(D^{\gamma_{t}}(G)\right)$.

COROLLARY 5.2. Let $R$ be a semi-prime left Goldie ring and $G$ a torsion-free Ore group. Then $R^{\gamma}(G)$ is semi-prime left Goldie.

Before the main theorem of this section we require the following lemma, the proof of which is routine.

LEMma 5.3. Let $G$ be a group and let $S$ be the set of all periodic elements of $G$. Then

(i) $C_{G}(S) \triangleleft G$,

(ii) $|S|<\infty \Rightarrow S \triangleleft G$,

(iii) $|S|<\infty \Rightarrow\left|G: C_{G}(S)\right|<\infty$. 
THEOREM 5.4. Let $R$ be a semi-prime left Goldie ring and let $G$ be an Ore group such that the set $S$ of all periodic elements of $G$ is finite with $|S|$ regular in $R$. Then $R^{\gamma}(G)$ is semi-prime left Goldie.

Proof. Let $\bar{C}_{G}(S)=\left\{g \in C_{G}(S): \gamma(g, s)=\gamma(s, g)\right.$ for all $\left.s \in S\right\}$. By Lemma 2.5, $\left|C_{G}(S): \bar{C}_{G}(S)\right|<\infty$. Hence, since $\left|G: C_{G}(S)\right|<\infty,\left|G: \bar{C}_{G}(S)\right|<\infty$. Also, by Theorem 2.7, $P R^{\gamma}(G)=0$ and so, by Lemma 4.1, it is sufficient to prove that $R^{\gamma}\left(\bar{C}_{G}(S)\right.$ ) is semi-prime left Goldie. Let $C=\bar{C}_{G}(S) \cap S$. Then $C$ is a central subgroup of $\bar{C}_{G}(S)$ and, since $C \subseteq S$, $\bar{g} \bar{c}=\bar{c} \bar{g}$ for all $g \in \bar{C}_{G}(S), c \in C$. Therefore, by Theorem 2.1 , we may construct a twisted group ring of $\bar{C}_{G}(S) / C$ over $R^{\gamma}(C)$ with twist $\delta$ (say) such that

$$
R^{\gamma}\left(\bar{C}_{G}(S)\right) \cong\left[R^{\gamma}(C)\right]^{\delta}\left(\bar{C}_{G}(S) / C\right)
$$

But, since $|C|<\infty$ and $|C|$ is regular in $R, R^{\gamma}(C)$ is semi-prime left Goldie (Lemma 4.1). Also, since $G$ is an Ore group, $\bar{C}_{G}(S)$ is an Ore group. Then, since $C$ is the set of periodic elements of $\bar{C}_{G}(S)$ and $C$ is finite, $\bar{C}_{G}(S) / C$ is a torsion-free Ore group. It now follows from Corollary 5.2 that $\left[R^{\gamma}(C)\right]^{s}\left(\bar{C}_{G}(S) / C\right)$ is a semi-prime left Goldie ring. That is, $R^{\gamma}\left(\bar{C}_{G}(S)\right)$ is semi-prime left Goldie and hence $R^{\gamma}(G)$ is also semi-prime left Goldie.

Definitions. If $\mathscr{X}$ is a class of groups, $L \mathscr{X}$ is the class of locally $\mathscr{X}$-groups consisting of all groups $G$ such that every finite subset of $G$ is contained in a $\mathscr{X}$-subgroup.

$\mathscr{X}$ is called a local class if $L \mathscr{X}=\mathscr{X}$. [10, part 1 p. 5, part 2 p. 93].

THEOREM 5.5. The class $\mathscr{C}$ of Ore groups is a local class.

Proof. Let $G \in L \mathscr{C}$. Let $S$ be a finite subset of $G$ and let $H=\langle S\rangle$. Since $G \in L \mathscr{C}$, there exists $K \in \mathscr{C}$ such that $S \subseteq K$. Then $H \leqq K$ and so $H \in \mathscr{C}$. From this it is clear that $L \mathscr{C}$ satisfies (i) and (ii) of the definition of an Ore group. We must now prove that if $G \in L \mathscr{C}$ is torsion-free and $D$ is a division ring then $D^{\gamma}(G)$ is an Ore domain. To prove this we show that

(a) $x y=0$ if and only if $x=0$ or $y=0\left(x, y \in D^{\gamma}(G)\right)$;

(b) given $x, y \in D^{\gamma}(G)$, there exist $x^{\prime}, y^{\prime} \in D^{\gamma}(G)$ such that $x^{\prime} x=y^{\prime} y$.

Let $x, y \in D^{\gamma}(G)$; then there exists a finitely generated subgroup $H$ such that $x, y \in D^{\gamma}(H)$. Then $H \in \mathscr{C}$ so that $H$ is a torsion-free Ore group and $D^{\gamma}(H)$ is an Ore domain. Now, since $x, y \in D^{\gamma}(H)$, they satisfy conditions (a) and (b). Hence $D^{\gamma}(G)$ is an Ore domain. We have shown that $L \mathscr{C}$ satisfies (i), (ii) and (iii) of the definition of $\mathscr{C}$. Hence $L \mathscr{C} \subseteq \mathscr{C}$ and so $L \mathscr{C}=\mathscr{C}$.

COROLlARY 5.6. Let $G$ be a locally nilpotent group (locally FC group); then $G$ is an Ore group.

THEOREM 5.7. Let $G$ be a locally nilpotent (locally $F C$ ) group. Then $R(G)$ is semi-prime left Goldie if and only if

(i) $R$ is semi-prime left Goldie, and

(ii) the subgroup $S$ of all periodic elements of $G$ is finite with $|S|$ regular in $R$. 
Proof. That (i) and (ii) are sufficient for $R(G)$ to be semi-prime left Goldie follows from Theorem 5.4.

Conversely, let $R(G)$ be semi-prime left Goldie. It is not hard to show that $R$ must be a left Goldie ring. Then, by Theorem $\mathrm{A}$ and the fact that the set of periodic elements of a locally nilpotent (locally $F C$ ) group is a locally finite subgroup, it follows that (i) and (ii) hold true.

THEOREM 5.8. Let $G$ be a group and let $H \triangleleft G$ be such that $H$ is periodic and $G / H$ is an Ore group. Then $G$ is an Ore group.

Proof. Let $\mathscr{X}=\{G: G$ has a periodic normal subgroup $H$ with $G / H$ an Ore group $\}$. Clearly $\mathscr{C} \subseteq \mathscr{X}$. We shall prove that $\mathscr{X}$ satisfies the definition of $\mathscr{C}$ and hence that $\mathscr{X}=\mathscr{C}$.

Let $G \in \mathscr{X}$ with $H \triangleleft G$ such that $H$ is periodic and $G / H \in \mathscr{C}$.

(i) If $K \leqq G$, then $K \cap H$ is a periodic normal subgroup of $K$. Also $K /(K \cap H) \cong$ $K H / H \leqq G / H \in \mathscr{C}$. Hence $K /(K \cap H) \in \mathscr{C}$ and it follows that $K \in \mathscr{X}$.

(ii) Let $K \triangleleft G,|K|<\infty$. Now $H K / K \cong H /(H \cap K)$ is a periodic normal subgroup of $G / K$. Also $(G / K) /(H K / K) \cong(G / H) /(H K / H)$ which belongs to $\mathscr{C}$, since $G / H \in \mathscr{C}$ and $H K / H \cong$ $K /(H \cap K)$ is a finite normal subgroup of $G / H$. Hence $G / K \in \mathscr{X}$.

(iii) If $G$ is torsion-free, then $H$ is trivial and hence $G \in \mathscr{C}$.

We have shown that $\mathscr{X}$ satisfies conditions (i), (ii) and (iii) of the definition of $\mathscr{C}$. Hence $\mathscr{X}=\mathscr{C}$.

\section{REFERENCES}

1. I. G. Connell, On the group ring, Canad.J. Math. 15 (1963), 650-685.

2. A. W. Goldie, Structure of noetherian rings, Lectures on rings and modules, Lecture Notes in Mathematics No. 246 (Springer-Verlag, 1972).

3. I. N. Herstein, Noncommutative rings, Carus Mathematical Monographs (Math. Assoc. of America, 1968).

4. A. Horn, Gruppenringe fastpolyzyklischer Gruppen und Ordnungen in Quasi-FrobeniusRingen, Mitt. Math. Sem. Giessen 100 (1973).

5. S. A. Jennings, The group ring of a class of infinite nilpotent groups, Canad. J. Math. 7 (1955), 169-187.

6. J. Lambek, Lectures on rings and modules (Blaisdell, 1966).

7. D. S. Passman, Nil ideals in group rings, Mich. Math.J. 9 (1962), 375-384. 409-437.

8. D. S. Passman, Radicals of twisted group rings, Proc. London Math. Soc. (3) 20 (1970), 633-651.

9. D. S. Passman, Radicals of twisted group rings II, Proc. London Math. Soc. (3) 22 (1971),

10. D. J. S. Robinson, Finiteness conditions and generalised soluble groups, Parts 1,2 Ergebnisse der Mathematik und ihrer Grenzgebiete Vol. 62, 63 (Springer-Verlag, 1972).

11. J. C. Robson, Artinian quotient rings, Proc. London Math. Soc. (3) 17 (1972), 600-616.

12. P. F. Smith, Quotient rings of group rings, J. London Math. Soc. (2) 3 (1971), 645-660.

\section{Mathematics Department}

UNIVERSITY OF ABERDEEN

ABERDEEN AB9 2UB 